\title{
LABORATÓRIO DE DESEMPENHO ESPORTIVO LADESP
}

\author{
Maria Augusta Peduti Dal'Molin KISS \\ Maria Tereza Silveira BÖHME**
}

Este artigo será apresentado de forma sucinta em três tópicos relacionados à área de estudo: caracterização, perspectivas e atuação.

\section{CARACTERIZAÇÃo}

Desempenho esportivo é a execução ótima de uma tarefa de movimento (Schüler Duden-Sport, 1987), sendo um componente integral do esporte, em todos os seu níveis. Ele é analisado de acordo com uma visão sistêmica de processo e de produto (Kiss, 1987) em diferentes grupos etários, incluindo desde atletas de alto rendimento até esportistas portadores de necessidades especiais.

O desempenho esportivo é um fenômeno complexo, devido os seus vários aspectos condicionantes. $\mathrm{O}$ desempenho, ou "performance", conforme o modelo de Malina (1980), foi considerado como um fenômeno expresso em três dimensões: orgânica, motora e cultural. Em termos conceituais é considerado de forma diferente nas várias áreas de estudo e pesquisa da Ciência do Esporte (Beyer, 1987; Carl, 1988; Friedrich, Grosser \& Preising, 1988; Haag, 1994; Martin, Klaus \& Lehnertz, 1991; Schüler Duden-Sport, 1987), a saber: antropologia, filosofia, medicina esportiva (fisiologia e clínica), biomecânica, psicologia, sociologia, história e ciência/teoria do treinamento.

Schnabell, Harre \& Borde (1994) consideram o desempenho esportivo, o treinamento esportivo e a competição esportiva como os três componentes principais no estado científico atual da Ciência do Treinamento; as relações existentes entre eles são apresentadas graficamente na FIGURA 1.

Na área da Ciência/Teoria do Treinamento, segundo Friedrich et alii (1988), "desempenho é 0 conjunto de processos e resultados de uma ação esportiva, orientado segundo uma dada norma social" Já para Martin et alii (1991), "desempenho esportivo é o resultado de uma ação esportiva, que encontra a medida de sua manifestação especialmente nos esportes de competição, a qual tem suas ações de movimento coordenadas por regras pré-estabelecidas"

De acordo com essas conceituações de desempenho esportivo, conclui-se que o mesmo é definido diferentemente pelos autores da área de treinamento esportivo. Assim, o primeiro autor considera o desempenho esportivo como processo e resultado; já para o segundo, desempenho esportivo refere-se somente ao resultado obtido na ação esportiva, pois o mesmo assume que os problemas principais a serem estudados e pesquisados pela teoria e considerados na prática

\footnotetext{
" Coordenadora do Laboratório de Desempenho Esportivo da Escola de Educação Física e Esporte da Universidade de São Paulo.

** Vice-Coordenadora do Laboratório de Desempenho Esportivo da Escola de Educação Física e Esporte da Universidade de São Paulo.
} 
do treinamento são a análise do resultado e o prognóstico do resultado.

O desempenho esportivo depende de normas sociais estabelecidas, o que significa que o(a) atleta carrega consigo a sua personalidade, o seu meio social, as suas fraquezas e as suas forças humanas. $\mathrm{O}$ treinador também é confrontado com expectativas sociais: em relação ao seu comportamento e em relação ao que se espera como desempenho esportivo na escala de valores da sociedade; essas relações são representadas na FIGURA 2, modificada de Böhme (1994).

Desempenho Esportivo
- Ação de desempenho
- Pressupostos de desempenho
- Desenvolvimento da
Capacidade de desempenho
(do estado de desempenho)
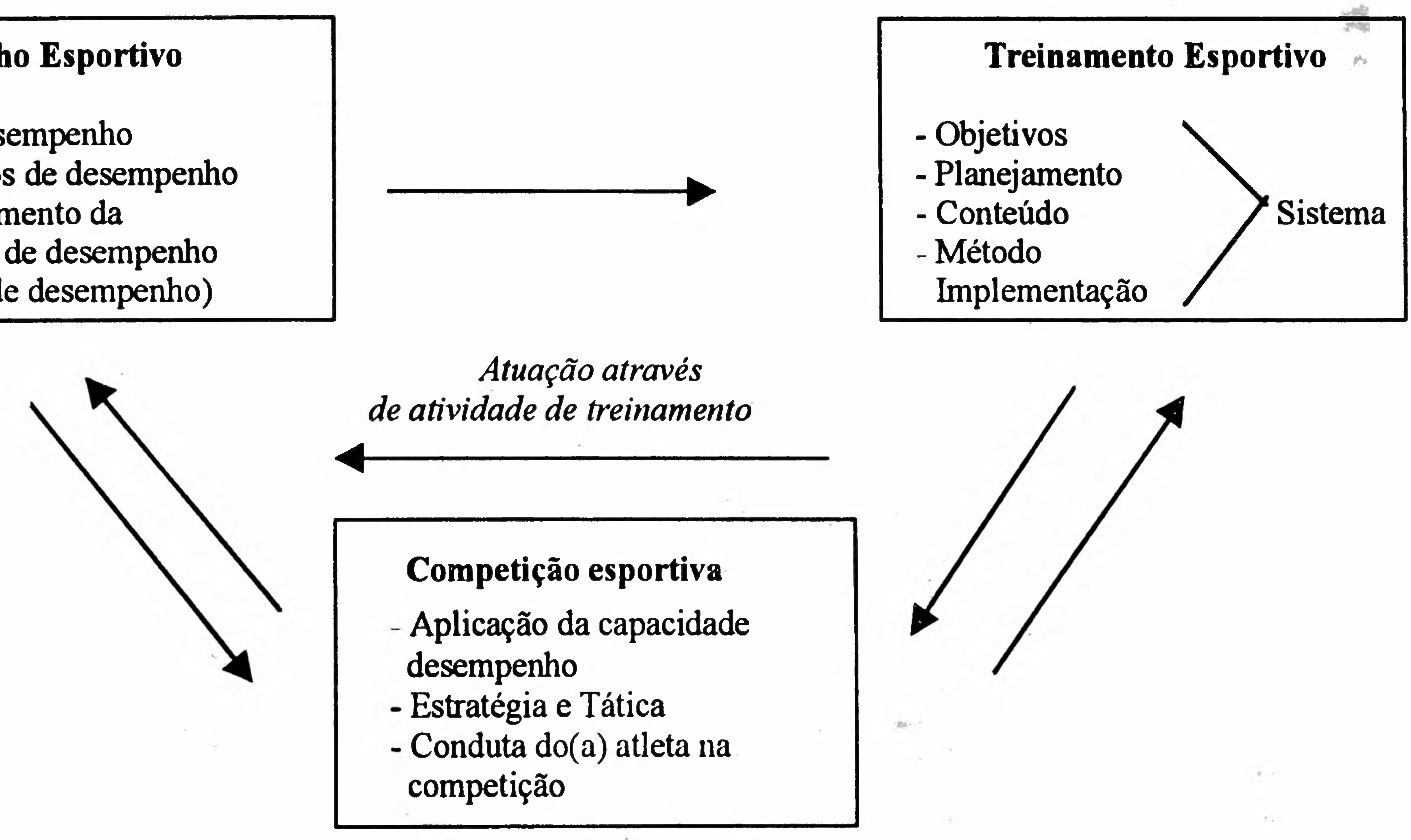

FIGURA 1 - Relações entre os componentes no estudo da teoria de treinamento (modificado de Böhme, 1994).

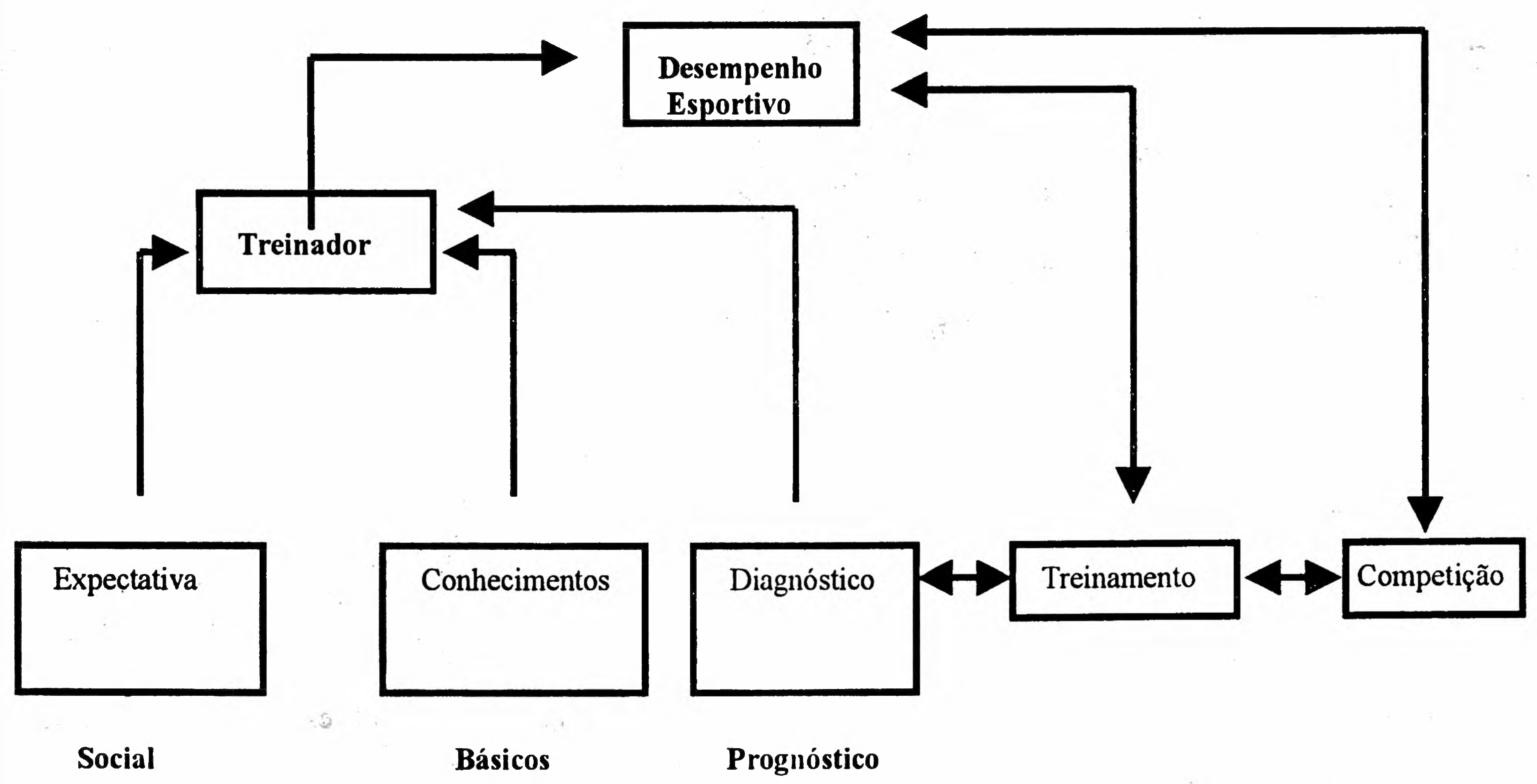

FIGURA 2 Relações gerais do desempcnho esportivo (modificado de Böhme, 1994). 
$\mathrm{Na}$ literatura alemã referente à Ciência do Treinamento, são encontrados diferentes modelos elaborados com o objetivo de representar a estrutura do desempenho esportivo (Carl, 1988; Friedrich et alii, 1988; Martin et alii, 1991; Röthig \& Größing, 1990), os quais foram descritos por Böhme (1994) assim como por outros autores, tais como Schnabell et alii (1994) e Weineck (1996).

O desempenho esportivo, conforme proposto inicialmente por Carl (1988), adaptado e atualizado por Böhme (1994), é analisado em relação às condições pessoais e aos fatores limitantes.
As condições pessoais são os pressupostos individuais do desempenho esportivo, expressas através da condição/aptidão e influências do meio ambiente; o seu estado é, em parte, determinado pelo treinamento. Algumas variáveis tem 0 seu desenvolvimento limitado por características genéticas e/ou do meio ambiente. As regras de treinamento pressupõem o conhecimento da treinabilidade de cada condição de forma individualizada. Na FIGURA 3 é apresentado o esquema das condições pessoais internas tanto diretas, quanto indiretas.

\section{Desempenho esportivo}

\section{$\uparrow$}

Condição fisica

Força Resistên- Veloci- Flexibi

cia dade lidade

Estrutura e Composição Corporal
Técnica dos movimentos

Capacidades Habilidades de

coordenativas movimento
Tática esportiva

Capacidade para resolver problemas adequadamente

Condições pessoais internas diretas de desempenho

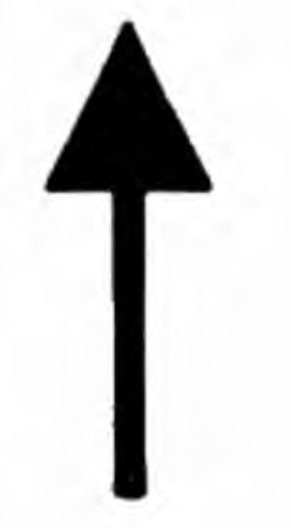

\begin{tabular}{|c|c|c|c|c|c|c|}
\hline \multicolumn{2}{|c|}{$\begin{array}{c}\text { Capacidades } \\
\text { orgânicas }\end{array}$} & $\begin{array}{l}\text { Capacidades } \\
\text { motoras }\end{array}$ & \multicolumn{2}{|c|}{$\begin{array}{l}\text { Capacidades } \\
\text { cognitivas }\end{array}$} & \multicolumn{2}{|c|}{$\begin{array}{l}\text { Capacidades } \\
\text { afetivas }\end{array}$} \\
\hline $\begin{array}{c}\text { Sistema } \\
\text { ósteo muscular }\end{array}$ & $\begin{array}{c}\text { Sistema } \\
\text { hemodinâmico }\end{array}$ & $\begin{array}{l}\text { Sistema } \\
\text { respiratório }\end{array}$ & $\begin{array}{l}\text { Sistema } \\
\text { cardio-circulatório }\end{array}$ & $\begin{array}{l}\text { Sistema } \\
\text { hormonal }\end{array}$ & $\begin{array}{l}\text { Sistema } \\
\text { digestivo }\end{array}$ & $\begin{array}{l}\text { Sistema } \\
\text { nervoso }\end{array}$ \\
\hline
\end{tabular}

Condições pessoais internas indiretas de desempenho

FIGURA 3 - Esquema das condição pessoais de desempenho e sucesso esportivo (modificado de BÖHME, 1994). 
As condições pessoais internas indiretas de desempenho são compostas pelos sistemas, órgãos e tecidos, formando a base de sustentação anatômica e das capacidades funcionais, motora, cognitiva e afetiva; essas, por sua vez, sustentam as condições pessoais internas diretas: a condição biológica (englobando aspectos estruturais, de composição corporal, e funcionais, de acordo com conceito de cineantropometria Kiss, Böhme \& Regazzini, 1999), a técnica do movimento e a tática esportiva; cada uma dessas resulta da atuação e interação, de proporções complexas, das condições pessoais indiretamente observáveis.

A característica principal que diferencia as condições pessoais diretamente observáveis das indiretamente observáveis, é a dependência das primeiras em relação às últimas: nas condições pessoais diretamente observáveis existe a atuação de complexos componentes das capacidades de desempenho psíquicas e corporais; nas condições pessoais indiretamente observáveis é mais simples a distinção das funções específicas de cada variável, embora também exista grande relacionamento entre as mesmas.

As condições limitantes do desempenho esportivo subdividem-se em pessoais (relativa ao próprio atleta), e sociais (relativas às condições materiais e sociais do seu meio ambiente). As primeiras englobam (realizando uma adaptação de Böhme, 1994): escola/estudo ou profissão, amigos, lazer e família. As condições limitantes sociais são: atitudes da sociedade para o desempenho esportivo; valor da modalidade esportiva no sociedade; meios financeiros disponíveis; e treinador com suas características de formação e nível de conhecimento.

Considerando-se as características apresentadas, o objetivo do LADESP é o estudo do desempenho esportivo considerado do ponto de vista sistêmico, relacionado às seguintes áreas da Ciência do Esporte: cineantropometria, medicina esportiva e treinamento fisico.

\section{PERSPECTIVAS}

$\mathrm{Na}$ análise micro do produto, muitas são as questões que se colocam: desde a adequação de instrumentos para estudos em campo (quadra, pista), bem como a determinação de erros de várias metodologias morfológicas e funcionais em laboratório.
Questões importantes encontram-se relacionadas a sobrecargas anaeróbias, analisadas mediante estímulos únicos ou repetidos, caracterização de testes intermitentes com cargas cuja intensidade seja supra segundo limiar de lactato e supra consumo máximo de oxigênio; nestas últimas, o componente aeróbio tem sido pouco estudado.

Houve renovado interesse no estudo de variáveis que pudessem diagnosticar fadiga crônica subclínica (evidenciando aumento de atividade simpática), tais como tempo de reação e comportamento de variabilidade de freqüência cardíaca

Também tem sido dada grande importância ao aspecto da quantificação objetiva das sobrecargas de treinamento, imprescindível para análise do processo, relacionadas a componentes aeróbio e anaeróbio.

Estão sendo revistas tanto as metodologias para estudos morfológicos e de composição corporal, quanto as análises segmentares e de proporções. As medidas antropométricas, de densimetria, pletismografia corporal e de rádio-diagnóstico estão sendo confrontadas para 0 estabelecimento de novo "padrão ouro"

A análise macroscópica do desempenho esportivo envolve a interrelação de fatores bio-psico-sócio-culturais (Burwitz, Moore \& Wilkinson, 1994), por meio de análises multivariadas utilizadas em trabalhos interdisciplinares. Um caso especial corresponde às pesquisas temáticas em modalidades específicas, em que se analisam desde aspectos morfológicos e funcionais até psicológicos (incluindo controle motor) e sociais, relacionando-os ao desempenho.

Outros aspectos importantes são estudados no treinamento a longo prazo (Bar-Or, 1996). Inclui-se nesse tópico o estudo dos valores prognósticos, considerando-se a estabilidade ("tracking") de diferentes variáveis, em função de crescimento, desenvolvimento e treinamento.

\section{ATUAÇÃo NO CAMPO CIENTÍfico E ASSISTENCIAL}

\section{Científico}

Esse tópico será analisado na perspectiva dos últimos 10 anos, em conseqüência das atividades da Coordenadora terem sido dirigidas com o objetivo acima apresentado, a 
partir de 1990, quando passou a integrar o Departamento de Esporte, organizando o Laboratório de Pesquisas em Esporte (LAPAE).

A seguir são apresentadas as Linhas de Pesquisa desenvolvidas e os professores do Departamento que nelas atuam:

\section{$>$ Avaliação de resistêncialendurance}

Maria Augusta P. Dal'Molin Kiss, Maria Tereza Silveira Böhme.

Análise das características funcionais e metabólicas aeróbias em praticantes de diferentes atividades esportivas, sua relação com treinamento, saúde, crescimento e desenvolvimento, de acordo com modelos máximos e submáximos, em situações de campo e de laboratório. Componente aeróbio em somatório de cargas supra máximas.

\section{Deteção, seleção e promoção de Talento Esportivo (TE)}

Maria Augusta P. Dal'Molin Kiss, Maria Tereza Silveira Böhme, Antônio Carlos Mansoldo.

Dentro de treinamento a longo prazo, são analisados aspectos interdisciplinares ou específicos e suas relações com crescimento e desenvolvimento.

\section{$>$ Potência e capacidade anaeróbias}

Maria Augusta P. Dal'Molin Kiss, Maria Tereza Silveira Böhme, Mauro G. Carvalho. Caracterização de potência e capacidade anaeróbia em laboratório e em campo.

\section{$>$ Condicionamento fisico esportivo}

José Alberto de Aguillar Cortez, Antônio Carlos Mansoldo.

Estudo de variáveis (capacidades) condicionais e coordenativas, métodos e meios de treinamento e dos fatores intervenientes.

$>$ Esporte e portadores de necessidades especiais

Elizabeth Matos.

Analisa características aeróbias e anaeróbias em esportistas portadores de necessidades físicas especiais.

$>$ Desenvolvimento clínico-cardiológico em crianças e adolescentes

Marcelo Regazzini, Maria Augusta Peduti Dal'Molin Kiss

Análise de aspectos cardiológicos clínicos e laboratoriais de crianças e adolescentes submetidos a treinamento a longo prazo.

\section{$>$ Composição corporal de esportistas}

Antônio Carlos Mansoldo, Emédio Bonjardim, Elizabeth Matos, Maria Tereza Silveira Böhme, Maria Augusta Peduti Dal'Molin Kiss.

Análise da massa muscular, óssea e de gordura de esportistas de diferentes modalidades e de portadores de necessidades especiais, a partir de densimetria e de equações genéricas e/ou específicas.

\section{Assistencial}

O LADESP tem atendido a comunidade, de acordo com tecnologias acima descritas, em especial a partir de 1997, quando passamos a fazer parte da Rede de Centros de Excelência Esportiva do Instituto para Desenvolvimento de Esporte (INDESP), Ministério dos Esportes e Turismo como CENESP-USP. Conforme consta em nosso relatório para esse órgão em 1998, a síntese dos procedimentos consta nas FIGURAS 4 e 5. 


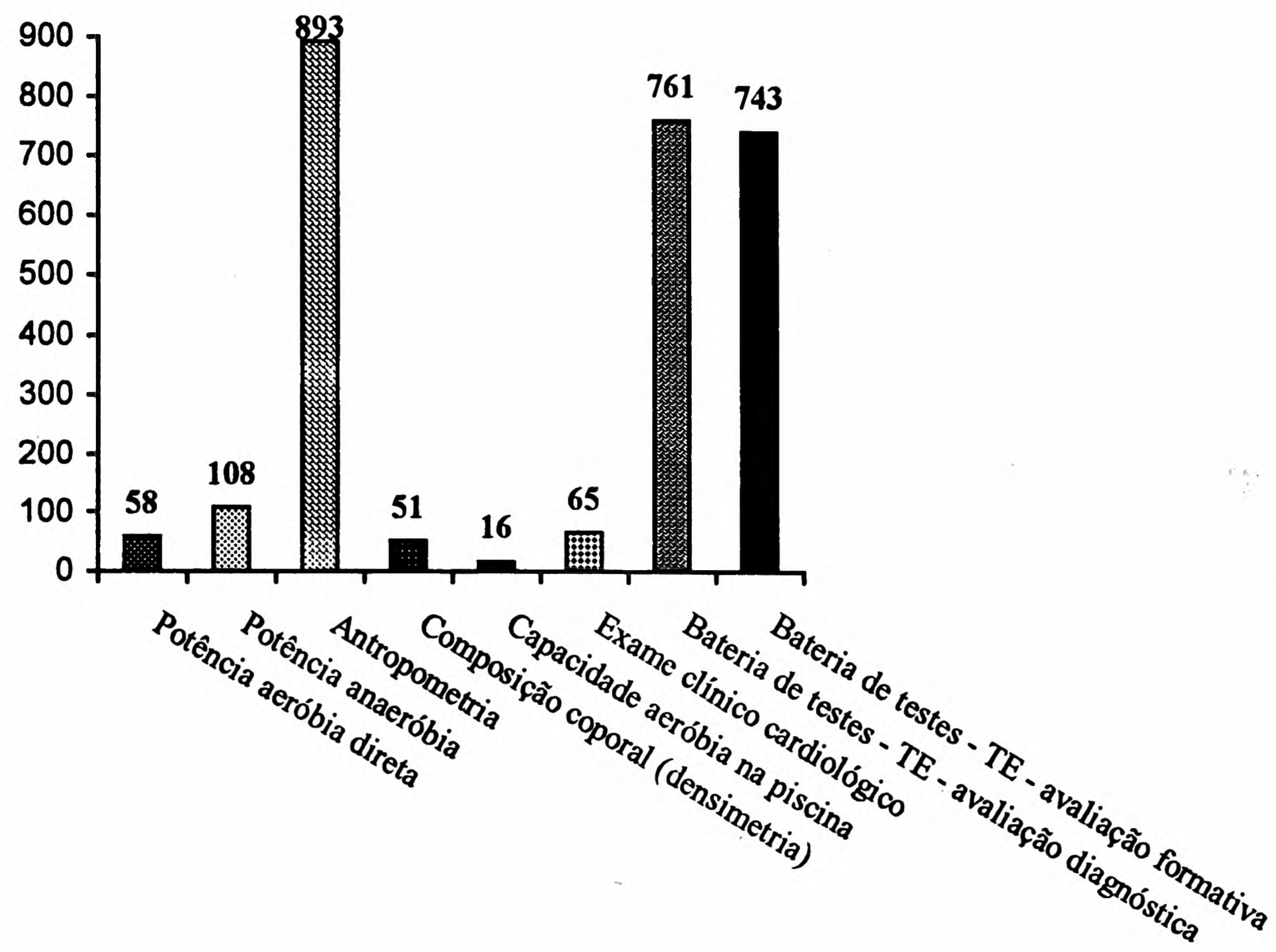

FIGURA 4 - Extensão de serviços à comunidade - procedimentos.

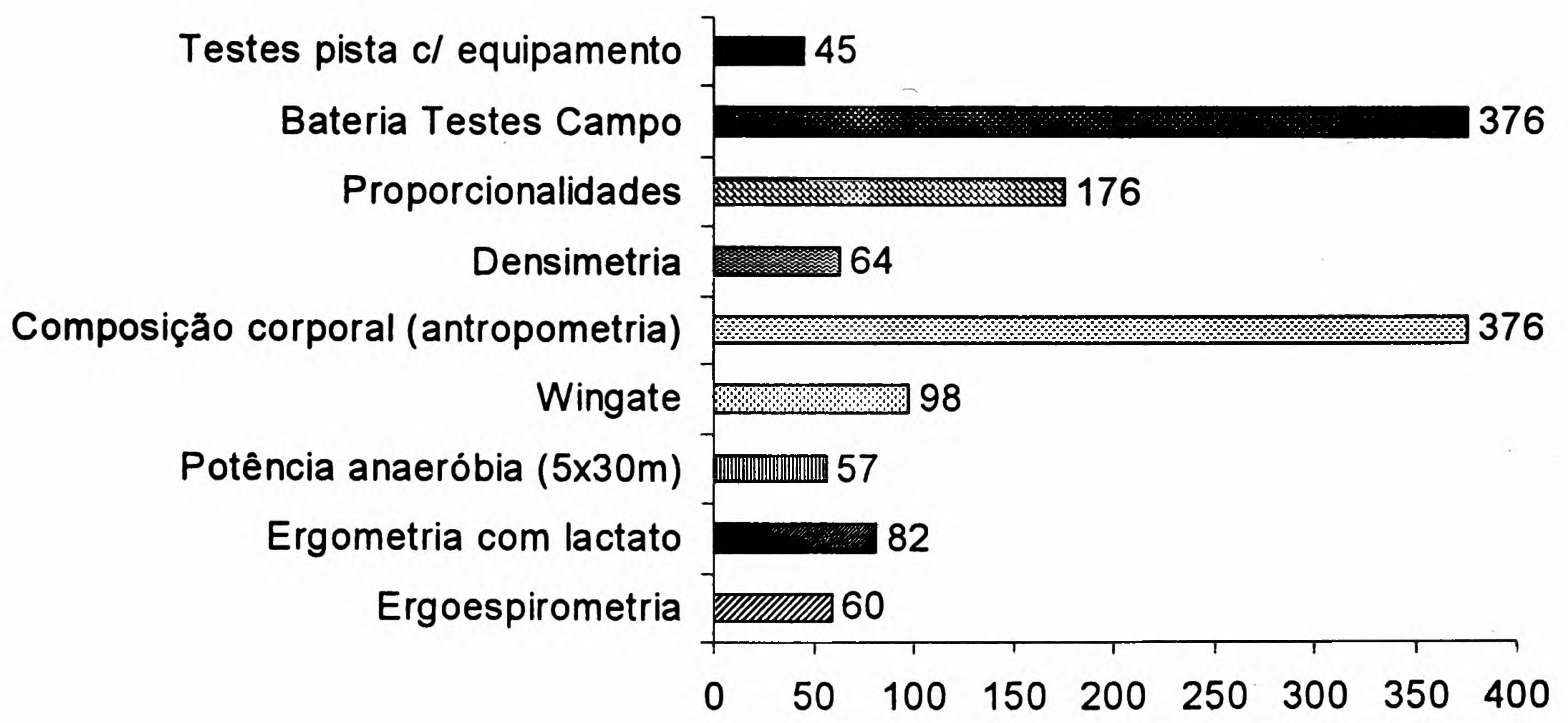

FIGURA 5 - Procedimentos 1998/1999. 


\section{NOTA}

Este artigo teve a colaboração de Prof.Dr. Antônio Carlos Mansoldo, Prof.Ms. Emédio Bonjardim, Prof.Ms. Marcelo Regazzini, Prof.Ms. Elizabeth Matos e Mestrandos Valéria Martim, Roberto Costa.

\section{REFERÊNCIAS BIBLIOGRÁFICAS}

BARBANTI, V.J. Treinamento físico: bases científicas. São Paulo, CLR Balieiro, 1986.

BAR-OR, $O$. The child and adolescent athlete. Oxford, Blackwell Science. 1996.

BEYER, E., ed. Wörterbuch der Sportwissenschaft. Schorndorf, Karl Hofmann, 1987.

BÖHME, M.T.S. Talento esportivo I: aspectos teóricos. Revista Paulista de Educação Física, v.8, n.2, p.90100, 1994.

BURWITZ, L.; MOORE, P.M.; WILKINSON, D.M. Future directions for performance-related sports science research: an interdisciplinary approach. Journal of Sports Sciences, v.12, n.1, p.93-109, 1994.

CARL, K. Talentsuche, Talentauswahl und Talentförderung. Schorndorf, Karl Hofmann, 1988.
FRIEDRICH, E.; GROSSER, M.; PREISING, R. Einführung in die Ausbildung von Trainernan der Trainerakademie. Schorndorf, Karl Hofmann, 1988.

HAAG, $\mathrm{H}$. Theoretical foundation of sport science as a scientific discipline: contribuition to a philosophy (meta-theory) of sport science. Schorndorf, Karl Hofmann, 1994.

KISS, M.A.P.D.M. Avaliação em educação física: aspectos biológicos e educacionais. São Paulo, Manole, 1987.

KISS, M.A.P.D.M.; BÖHME, M.T.S.; REGAZZINI, M. Cineantropometria. In: GORAYHEB, N.; BARROS NETO, T.L. O exercício: preparação fisiológica, avaliação médica, aspectos especiais e preventivos. São Paulo, Atheneu, 1999.

MALINA, R.M. A multidisciplinary approach to physical performance. In: OSTYN, M.; BEUNEN, G.; SIMONS, J., eds. Kinanthropometry II. Baltimore, University Park Press, 1980. p.33-68.

MARTIN, D.; KLAUS, C.; LEHNERTZ, K. Handbuch Trainingslehre. Schorndorf, Karl Hofmann, 1991.

SHEPHARD, R.J. Questioning our research assumptions. Adapted Physical Activity Quarterly, v.15, n.3, p.222-35, 1998.

SCHNABELL, G.; HARRE, D.; BORDE, A., eds. Trainingswissenschaft. Leistung - Training Wettkampf. Berlin, Sportverlag, 1994.

SCHÜLER DUDEN. Der Sport. Mannheim, Meyers Lexikonverlag, 1987.

WEINECK, J. Optimales training. Erlangen, Perimed Fachbuch, 1996. 